\title{
RESENTIMIENTO: NEUROCIENCIAS APLICADA AL PSICOANÁLISIS, UN ENSAYO TEÓRICO TRANSDISCIPLINARIO
}

\section{ENSAYO TEÓRICO}

MELLO, Paulo de ${ }^{1}$, BERTINI, Edna², FREIRE, Lázaro Luiz Trindade ${ }^{3}, J^{2}$ ACINTO, Débora Damasceno ${ }^{4}$, BORGES, Tássia Monteiro ${ }^{5}$

MELLO, Paulo de. Et al. Resentimiento: neurociencias aplicada al psicoanálisis, un ensayo teórico transdisciplinario. Revista Científica Multidisciplinar Núcleo do Conhecimento. Año 06, Ed. 11, Vol. 06, págs. 133-146. Noviembre de 2021. ISSN: 2448-0959, Enlace de acceso:

https://www.nucleodoconhecimento.com.br/psicologia-es/neurociencias-aplicada,

DOI: 10.32749/nucleodoconhecimento.com.br/psicologia-es/neurociencias-aplicada

\section{RESUMEN}

Objetivamos con este articulo presentar una concepción transdisciplinar de la relación entre neurociencia y psicoanálisis, sobre todo la kleiniana, en el campo de los epifenómenos ligados al resentimiento, su significado y mecanismos fundamentales de naturaleza psicoanalítica y biológica. El artículo es resultado de un

\footnotetext{
${ }^{1}$ Doctorando en Psicología Clínica de la Universidad de Ciencias Empresariales y Sociales (UCES/Buenos Aires/Argentina); Máster en Psicología de la Salud por la Universidad Metodista de São Paulo (UMESP/SP/Brasil); Especialista en Medicina del Comportamiento por la Universidade Federal de São Paulo (UNIFESP/SP/Brasil); Neurólogo Clínico por la Universidade Federal de São Paulo (UNIFESP/SP/Brasil); Médico por la Universidad de Taubaté (UNITAU/SP/Brasil).

${ }^{2}$ Doctora en Farmacología por el Instituto de Ciencias Biomédicas (USP/SP/Brasil). Máster en Farmacología por el Instituto de Ciencias Biomédicas (USP/SP/Brasil). Especialista en Medicina del Comportamiento (UNIFESP/SP/Brasil). Psicoanalista capacitada en Psicoanálisis Transpersonal (IBT). Psicoanalista capacitada en Análisis Reichiana (IBAR).

${ }^{3}$ Psicoanalista formado en Psicoanálisis Transpersonal (IBT). Graduado en Ingeniería Eléctrica por la Pontifícia Universidade Católica (PUC/MG/Brasil). Graduado en Filosofía (UNIFAI/PUC/SP/Brasil).

${ }^{4}$ Psicoanalista formada por la Escuela de Psicoanálisis y Ciencias Humanas (EPCH/SP/Brasil). Especialista en Teoría Psicoanalítica (Sorbonne/Paris/França). Graduación en Filosofía por la Universidade de São Paulo (USP/SP/Brasil).

${ }^{5}$ Estudiante de maestría en Psicología Clínica de la Pontifícia Universidade Católica de São Paulo (PUC/SP/Brasil). Psicoanalista por la Escuela FACEI (SP/Brasil). Graduada en Letras por la Universidade de São Paulo (FFLCH/USP/SP/Brasil).
}

RC: 101104

Disponible: https://www.nucleodoconhecimento.com.br/psicologia-es/neurocienciasaplicada 
estudio teórico-cualitativo utilizándose como base la vivencia de los autores, algunos con más de 30 años de experiencia clínica en el área de la salud mental, psicoanálisis y neurociencia, sumado a una revisión bibliográfica que consta sobre todo de libros en el campo de la neuropsicoanálisis, análisis y psicoanálisis freudiana, kleiniana y junguiana, un total de 21 libros investigados, además de artículos en el campo de la neurociencia. Investigados en las bases de datos PubMed, Medline y Scielo en el periodo entre 2000 y 2020. El recorte epistemológico envuelve elementos como relación objetual, neurotransmisores, estructuras y circuitos neuronales envueltos en el fenómeno resentimiento. Fueron excluidos textos que se encontraban fuera del alcance cualitativo y transdisciplinar del estudio del texto. Utilizamos el método intuitivo-interpretativo cuya conclusión refuerza la viabilidad de la comprensión de fenómenos psicoanalíticos tal cual el determinismo psíquico y relaciones objetales vía intersección con mecanismos neurobiológicos que se desarrollan a través de operaciones mentales (mentalización), e intervención psicofarmacológica y de neuromodulación por estimulación magnética transcraniana, ampliando así el conocimiento sobre el asunto para las áreas en cuestión.

Palabras claves: resentimiento, neuropsicoanálisis, psicoanálisis, mentalización.

\section{INTRODUCCIÓN}

Basado en el deseo de ampliar el conocimiento en el área de investigación transdisciplinaria entre la neurociencia y el psicoanálisis, desarrollamos un estudio teórico-cualitativo apoyado por la experiencia clínica de los autores y revisión de textos, libros y artículos objetivando demostrar la viabilidad de desarrollarse estudios transdisciplinarios entre áreas aparentemente tan diferentes, no con el objetivo de validación, pero de ampliar la comprensión de cada área buscando puntos de convergencia entre ellas (TURATO, 2005; HULLEY et al., 2008).

Para este articulo optamos por estudiar el resentimiento, fenómeno común, responsable por el sufrimiento emocional y físico de muchos de aquellos que buscan atendimiento en los consultorios médicos, psicológicos y psicoanalíticos. 
Con base en esta pequeña introducción, entendemos, como parte de nuestra cosmovisión, que el yo - nuestra idea consciente de quienes somos o de lo que somos (HOLLIS, 1998) - surge como elemento virtual pues no puede ser palpado, ni sabemos de cual materia es hecho, entre tanto él aparece en algún momento en el periodo pre o neo natal con el objetivo casi obsesivo de buscar seguridad, de mantenerse vivo y de preservar la especie; algo que surge en el proceso evolutivo, similar a la garra del animal, sus dientes y velocidad. Visto de esta forma, el yo puede ser un representante fenomenológico fundamental de la vida física y del cuerpo físico, teniendo su origen natural en la función cerebral a medida que este mismo cerebro se desarrolla.

Visto de esta forma, el desarrollo del yo se realiza como un recurso del cuerpo físico, tal cual un software gerenciado por el cerebro. Su naturaleza o la naturaleza de su manifestación en el universo tiempo/espacio es bioeléctrica y bioquímica y la calidad y formas de expresión pueden ser modificadas, intensificadas o reprimidas por medio de la neuromodulación farmacológica y muy probablemente por la neuromodulación magnética por intermedio de la estimulación magnética transcraniana (TMS).

Nuestra revisión nos permite afirmar que existen sistemas neuronales razonablemente específicos implicados en el desarrollo y mejora del yo, de las relaciones objetales y de una agresividad innata. Estos datos corroboran con la teoría kleiniana.

\section{EL YO}

Como dijimos anteriormente, entendemos que el yo es básicamente la idea consciente de quienes somos o de qué somos (HOLLIS, 1998).

Al principio nos cabe exponer que el sistema meso límbico (dopaminérgico), y meso cortical, juntos, forman un sistema que impulsan al individuo a explorar su relación 
con el Otro en la figura de alguien fuera de él, un objeto parcial al principio (para satisfacer sus necesidades), y después, un objeto total (para satisfacer sus deseos).

Por esta razón, este sistema es fundamental para el desarrollo de la relación objetal, de la catéxia y para el mejoramiento de las funciones dichas yoicas. Hasta aquí entendemos que las relaciones objetales son fundamentales para la estructuración del yo; y para que exista relaciones objetales, hay la necesidad de que el cerebro desarrolle más que representaciones objetales, sino que sea también capaz de introyectar objetos esenciales o primordiales yendo más allá de las representaciones.

El yo, tal cual las demás estructuras de la $2^{a}$ tópica de Freud, se desarrolla, pues con el pasar de los años va siendo alimentado con un conjunto de informaciones que se acumulan día a día proveniente del universo interno y externo al individuo.

El universo externo al individuo es por él sentido a partir de la exterocepción (temperatura, dolor, tacto, visión, audición, paladar y olfato), de la propriocepción (percepción sensorial proveniente de los tendones, ligamentos, articulaciones y músculos) y de la interocepción (lo mismo para vasos sanguíneos y vísceras).

Es por medio de estos sentidos que el cerebro es alimentado por un incontable volumen de informaciones cuyo objetivo es el de mejorar la capacidad adaptativa del sujeto y sus relaciones objetales. Por medio de este proceso el yo desarrolla mecanismos o recursos que visa su sobrevivencia, son las dichas defensas del yo; recursos directamente dependientes del desarrollo y maduración del córtex prefrontal, pero no apenas esto.

\section{MENTALIZACIÓN Y RESENTIMIENTO}

La mentalización o tratamiento basado en la mentalización (TBM) tiene en cuenta la capacidad de tratar de comprender los mecanismos fenomenológicos, relacionales, representativos y biológicos de eventos de naturaleza mental, tales como: 
pensamientos, creencias, representaciones, sentimientos, ganas y deseos (BATEMAN; FONAGY, 2016).

El término Mentalización surgió con Peter Fonagy y otros autores en un intento de comprender mejor y buscar un modelo de enfoque psicoterapéutico para pacientes con trastorno borderline de la personalidad.

Según Cryan y Quiroga (2013), el vínculo afectivo seguro, especialmente en los primeros años de vida, es fundamental para el buen desarrollo de los patrones de funcionamiento mental que resultan en un sujeto más adaptado y con relaciones sociales más funcionales y por qué no decir, de aprendizaje. De esta manera, entendemos que el resentimiento puede ser el resultado del desarrollo de una rutina de operaciones mentales disfuncionales en entornos que no ofrecen al niño condiciones afectivas seguras.

La Mentalización requiere un análisis cuidadoso y contextualizado y su uso y desarrollo en el campo de la psicoterapia ha crecido en los últimos años, especialmente para el tratamiento del trastorno de estrés postraumático, trastornos de personalidad, drogadicción y trastornos alimentarios. Es necesario, en la mentalización, como enfoque terapéutico, evaluar cuidadosamente las rutinas de comportamiento, relaciones objetales y experiencias a las que el individuo estuvo expuesto y en el pasado se expuso. Recluta procesos cognitivos complejos y sus mecanismos ocurren principalmente en un nivel preconsciente (BATEMAN; FONAGY, 2016).

Se identificó 4 dimensiones diferentes para una mentalización, a saber: Mentalización automática versus mentalización controlada; Mentalización em si mismo versus mentalización en otros; Mentalización con miras a los aspectos internos versus externos; y Mentalización cognitiva versus afectiva (LIEBERMAN, 2007).

Entonces, considerando este modelo teórico y práctico con respecto al enfoque psicoterapéutico Mentalización, podríamos decir que el resultado de un conjunto de RC: 101104

Disponible: https://www.nucleodoconhecimento.com.br/psicologia-es/neurocienciasaplicada 
operaciones rutinarias y mentales de predominio automático, en sí mismo, con miras a los aspectos internos y afectivos, cuando en desequilibrio, puede dar lugar a este complejo fenómeno llamado resentimiento.

Por lo tanto, entendemos que el conocimiento psicoanalítico y de otras técnicas, combinado con una comprensión de los patrones mentales relacionados con el sujeto resentido, puede contribuir mucho al desarrollo y en la aplicación de herramientas psicoterapéuticas en su tratamiento.

\section{MODULAR EL YO ES POSIBLE POR LA PSICOFARMACOLOGÍA Y POR LA TMS}

Es hecho indiscutible que la suministración de antidepresivos de clases diversas es capaz de modular funciones cerebrales y proporcionar remisión de síntomas depresivos a partir de un complicado e intrincado mecanismo que envuelve receptores, recaptación de neurotransmisores, activación de transcripción de DNA por lo menos a medio plazo y finalmente neuroplasticidad.

Estudios demostraron que la neuroplasticidad es uno de los mecanismos de actuación de los antidepresivos, especialmente la neuroplasticidad envuelve la porción ventral del giro denteado de la formación hipocampal por aumento de neurotrofinas, particularmente BDNF. Los antidepresivos aumentan BDNF y por lo tanto promueven neuroplasticidad (YAMADA et al., 2013). Esta es una prueba indiscutible de que psicofármacos promueven mudanzas estructurales en sistemas neuronales.

Sabemos, de acuerdo con trabajos más recientes, que es posible reducir procesos cognitivos rumiativos, depresivos y auto despreciativos estimulándose la región dorso lateral del córtex prefrontal (CPFDL) o inhibido su área homologa contralateral utilizándose para esto la estimulación magnética transcraniana (TMS); y si tal neuromodulación es viable, modificar características, al menos manifiestas, que podríamos clasificar como yoicas, también es factible. 
Así, si podemos de hecho modificar su expresión, es porque el yo es pasible de ser influenciado por la química de los psicofármacos y por la aplicación de campos magnéticos con potencial para elevar o reducir la actividad de áreas específicas del cerebro.

\section{RESENTIMIENTO; AGRESIVIDAD DE NATURALEZA INNATA Y SISTEMA AVERSIVO CEREBRAL}

Hasta aquí, entendemos que todos estos modelos perceptivos - exterocepción, propiocepción e interocepción - son de naturaleza externa al proceso mental innato, incluso a los interoceptivos. Es un hecho que todas estas informaciones participan de la construcción del universo mental, pero tal vez Melanie Klein (1996) tenía razón cuando afirmo que al nacer somos dotados de mecanismos innatos que nos moviliza hacia adelante, por medio, no apenas de la nutrición y sexualidad, pero también por medio de la agresividad.

¿Qué evidencias científicas tenemos sobre esta afirmación, de que la agresividad es tan innata en cuanto a los metaprogramas neuronales responsables por la nutrición y reproducción?

Existe en el encéfalo un conjunto de estructuras y circuitos neuronales descrito como sistema aversivo cerebral (SAC). Este sistema es formado por la substancia periaquedutal, núcleos periventriculares del hipotálamo y núcleos centrales de la amígdala. Este sistema aversivo cerebral es modulado por el córtex prefrontal y es activado cuando el sujeto se siente amenazado física o emocionalmente. El alcohol, por ejemplo, inhibe el córtex prefrontal (CPF), lo que reduce la inhibición sobre el SAC facilitando comportamientos agresivos. Ja los medicamentos inhibidores selectivos de recaptura de serotonina elevan la actividad serotoninérgica en este circuito lo que resulta, podemos anticipar, en mayor control sobre el SAC, en la práctica, mayor tolerancia social (BRANDÃO et al., 2003)

RC: 101104

Disponible: https://www.nucleodoconhecimento.com.br/psicologia-es/neurocienciasaplicada 
En un artículo reciente (2018), publicado en la revista Nature Neuroscience, Stagkouraks et al, realizaron un estudio preclínico con roedores utilizándose da optogenética. Estudiaron mecanismos que regulan la agresión entre machos en la determinación de su posición social y apuntaron al núcleo hipotalámico pre mamilar ventral (PMv), como una estructura clave en la regulación de estímulos agresivos. De acuerdo con los autores, la estimulación del núcleo PMv desencadena comportamientos agresivos que se mantienen por semanas después de un breve estimulo. A este fenómeno el autor utilizo el término hysteresis.

Hysteresis es la tendencia que un sistema se manifiesta al conservar sus propiedades em la ausencia del estímulo que las produjo. Esto significa decir que, después de estimulado, el núcleo PMv, continúa disparando potenciales eléctricos por semanas cuyo representante fenomenológico, en este caso, es la agresividad. Considerando que el núcleo PMv es hipotalámico, podemos inferir que este sistema subcortical contenga un metaprograma comportamental innato para la agresividad ya presente y funcionante, tal vez, incluso antes del periodo neonatal. Este raciocinio corrobora para una mejor comprensión transdisciplinar sobre la afirmación, de acuerdo con la teoría kleiniana, de que la agresividad es tan innata en cuanto a otros informes de metaprogramas a la nutrición y sexualidad.

Hrabovszky et al. (2005), identificaron tres fenotipos neuronales en la estructura hipotalámica que están envueltas en la inducción de la agresividad, estos son: neuronas glutamatérgicas que coexpresan con la hormona liberadora de tireotrofina, neuronas glutamatérgicas sin hormonas liberadora de tireotrofina y neuronas GABA enérgicos dispersos entre las células glutametérgicas.

Estos son, hasta el momento, los elementos comunicadores y reguladores de la agresividad presentes en el hipotálamo y si el resentimiento fuera, de hecho, un acto agresivo y duradero contra sí mismo - pulsión de muerte - tal vez sean estos los neurotransmisores claves en el proceso de comunicación neural cuyo fenómeno final es lo que llamamos de resentimiento. 


\section{RESENTIMIENTO: MECANISMO COMPLEJO FUNCIONAL QUE BUSCA LA SOBREVIVENCIA DEL YO Y SE UTILIZA DE LA IDENTIFICACIÓN PROYECTIVA}

En la tentativa de conceptualizar el resentimiento vamos a apoyarnos en el libro Resentimiento: clínica psicoanalítica, da autora Maria Rita Kehl (2004).

Resentirse, de acuerdo con la autora, significa atribuir a otro la responsabilidad por lo que nos hace sufrir, pues a este Otro se imputa la culpa de aquella cuya expectativa no fue correspondida. Así, el resentido se siente incapaz de implicarse como sujeto de deseo e incluso que el resentimiento no sea un concepto psicoanalítico, parece que "(...) es una solución de compromiso entre el campo del narcicismo y el del Otro". (KEHL, 2004, p.11). De acuerdo con la autora, "(...) el resentido no es alguien incapaz de olvidarse o de perdonar, es uno que no quiere olvidarse o que quiere no se olvida, no perdonar, no dejar barato el mal que lo victimo". (KEHL, 2004, p.12).

Según Scheler (1958), el envenenamiento psicológico - resentimiento - es resultado de la reorientación de los impulsos agresivos para el yo, generando así la imposibilidad de alejarse de la memoria la actitud considerada la naturaleza de su resentimiento. La culpa es un sentimiento que el resentido busca atribuir al Objeto de su resentimiento; y en términos kleinianos esto sucede por medio de la identificación proyectiva (KLEIN, 1996). De este modo, el resentido busca hacer al Otro sufrir su sufrimiento, el sufrimiento de la culpa, del vacío, del abandono, de la negligencia y de la agresividad contra sí mismo.

De acuerdo con Rosenfeld (1971), el proceso de identificación proyectiva (IPry) se refiere a un proceso de cisión del yo más arcaico. Considerando que el proceso del resentimiento envuelve, dentro de otros mecanismos defensivos del yo, la IPry, podemos decir que el resentido, como resultado de esta cisión, proyecta objetos parciales representados por objetos amorosos o de odio para dentro de objetos externos (OExt).

RC: 101104

Disponible: https://www.nucleodoconhecimento.com.br/psicologia-es/neurocienciasaplicada 
Si estuviera en la posición Esquizoparanoide, el resentido percibiría el OExt como un perseguidor (ALMEIDA, 2003), pero si él estuviera en la posición Viscocárica (MELLO et al., 2017), el agresor será percibido como el objeto deseado faltante, aquel que abandono el resentido a la propia suerte, como si este fuese incapaz de sostenerse y de sobrevivir en la ausencia del OExt, como si este representase la madre o incluso al útero que nutre, o que debería, en su concepción inconsciente, nutrirlo. Se trata de un objeto aglutinado, que de acuerdo con Bleger (1977), comprende un complejo sistema simbólico donde objetos parciales (OPc) buenos y malos, se mezclan y no pueden ser discriminados (KLEIN, 1996).

De esta forma, no hay delimitación clara entre el yo y el no-yo, entre lo interno y lo externo; y por esta razón, el resentimiento se moviliza en bloque intentando arrastrar consigo la parte que considera como siendo suya, su agresor, dentro del proceso simbiótico, por esto es por lo que se utiliza de la IPry como recurso primordial.

Una condición fundamental para el desarrollo del resentimiento es que la persona resentida se sienta debilitada por su agresor, sumado a la negativa del sujeto a dejar la dependencia instituida por su agresor, la dependencia infantil de alguien que supuestamente debería protegerlo (KEHL, 2004) y ofrecerle el nurturance, continencia.

Así, en la infancia, el Otro, el agresor del resentido es representado por las figuras que tenían poder efectivo para proteger, premiar y punir al niño (KEHL, 2004).

Sobre una visión neo-kleiniana, la relación simbiótica y fusional, inherente a la posición Viscocárica, descrita inicialmente en español como Ghlischro-cárica por José Bleger (1977), explicitaría el resentimiento como un acto desesperado del yo de mantenerse fusionado al Objeto referencial, para quien, su demanda de amor, deseo de protección y continencia se ve proyectado y en el mínimo parcialmente frustrado (MELLO et al., 2017).

Mantenerse fusionado, para el resentido es una meta de sobrevivencia ya que cree no poder sustentarse y sobrevivir en la ausencia del objeto aglutinado. Cuando este RC: 101104

Disponible: https://www.nucleodoconhecimento.com.br/psicologia-es/neurocienciasaplicada 
sistema falla, surge la difusión y se instala profundo sentimiento de miedo, lo que denominamos de ansiedad defusional, un sentimiento de muerte inminente y desorganización del pensamiento sobre todo en su forma, manifiesto en la dimensión física como falta de aire, palpitación, dolor en el pecho, debilidad u hormiguero por el cuerpo, resultado de la desconstrucción del núcleo aglutinado y debilitamiento del yo.

En el ámbito de la neurociencia y de la salud mental este cuadro no solo se parece a lo que la psiquiatría y neurología denomina de trastorno de ansiedad del tipo pánico, pero de hecho es verdaderamente un síndrome del pánico, excepto en la situación por la cual tales síntomas se manifiestan como resultado de una ansiedad persecutoria, común a la posición esquizopanóide al revés de manifestarse como resultado de una ansiedad de defusión, objeto de nuestro estudio, común a la posición viscocárica (MELLO et al., 2017).

El síndrome de pánico surge por alteración en la sensibilidad excitatoria de una red de circuitos que envuelven estructuras como el córtex prefrontal, giro del cíngulo, ínsula, tálamo, amígdalas y proyecciones del núcleo amigdaloide para el tronco cerebral, hipotálamo y sistema septo-hipocampal. Los neurotransmisores más frecuentemente envueltos en esta modulación son la noradrenalina y la serotonina (MEZZASALMA et al., 2004).

Aparentemente el encéfalo entra en un estatus de emergencia y en seguida de urgencia, activando núcleos, circuitos y sistemas que generan un intenso estado de atención y entonces de desorientación en recurrencia a la activación noradrenérgica en las áreas citadas. Tal vez la naturaleza inmediata de esta disfunción sea la separación entre los objetos, de acuerdo con el autor Walter Trinca:

Percibiéndose sólo, toma consciencia de sus relaciones internas, lo que parece insoportable (...) perdura una sensación intima de que allá en el fondo, en vez de este núcleo del ser, hay la nada (...) si pudiese contar con la seguridad existencial de un contacto permanente con los fundamentos de sí mismo, el fóbico no tendría miedo de representarse solo y separado. (TRINCA, 1997, p.39).

RC: 101104

Disponible: https://www.nucleodoconhecimento.com.br/psicologia-es/neurocienciasaplicada 
Esta tal vez sea la naturaleza inmediata del miedo que surge cuando los recursos psicológicos y operaciones mentales utilizadas durante el complejo denominado como resentimiento no funcionan correctamente. Entre tanto es cierto que además de las dichas causas inmediatas, hay las causas mediatas y causas del rasgo.

Apuntamos como causas inmediatas, aquellas ligadas al desencadenamiento inmediato del miedo, a saber, la difusión, la separación entre los Objetos, como referido por Walter Trinca y José Bleger. Las causas mediatas - referidas como parte del determinismo psíquico del psicoanálisis - envuelven mecanismos epigenéticos resultado de la exposición del bebé y del infante a las relaciones con sus Objetos referenciales, sobre todo a la figura materna, el ambiente familiar y tal vez hasta el ambiente intrauterino. Ya las causas del rasgo son aquellas determinadas por rasgos genéticos de comportamiento.

Estudios preclínicos como lo realizado por Dias (2014), demuestran respuestas transgeneracionales relacionadas al miedo aprendido por hasta dos o más generaciones anteriores. No vemos un motivo para que este raciocinio no pueda ser utilizado para humanos y si de hecho se aplica a humanos, al miedo aprendido $o$ aprehendido por nuestros abuelos puede resultar en respuestas similares de miedo en las generaciones siguientes; por esto optamos por el termino causa del rasgo (causa de las características determinadas por la genética del sujeto), para la ansiedad defusional cuya naturaleza tenga su principio en una, dos o tal vez hasta más de tres generaciones anteriores.

\section{CONCLUSIÓN}

Es un hecho, que tanto el resentimiento, en términos de depresión o incluso el miedo derivado del fracaso de los recursos psicológicos que lo sustentan, son fruto de un complejo sistema, que, por los puntos de vista de la ciencia, engloba el encéfalo y circuitos estructurados, formateados y reformateados sobre influencia de la genética y epigenética; y que en el ámbito kleiniano puede ser entendido conforme José Bleger lo definió. 
Bleger, se refiere a este sistema como bastante precoz en la relación simbólica madre-hijo. Una desorganización en este sistema, de naturaleza neuronal u objetual trae a la superficie un complejo cuerpo de síntomas depresivos y de miedo al mismo tiempo.

Para José Bleger (1977), hay una parte no discriminada, ni diferenciada, que pertenece al yo del sujeto y de la realidad exterior que él llamo de objeto aglutinado. De acuerdo con Bleger, es un conglomerado con muchas experiencias frustrantes y también, gratificantes desde que se empieza la vida del bebé.

Comprendemos entonces que inferir al fenómeno resentimiento una única naturaleza, sea objetual, sea estructural - en términos biológicos - es en nuestra manera de ver, reduccionista. Creemos que una visión más sistémica y transdisciplinaria de estos fenómenos psicoanalíticos y biológicos, eleve y mucho nuestra capacidad de intervención en la búsqueda de un deshecho más adecuado en dirección al desarrollo humano a partir del ser; sea por medio de la comprensión y manipulación del setting terapéutico y de las relaciones transferenciales y contratransferenciales, sea incluso que concomitante, a través de la neuromodulación cuidadosa con psicofármacos o con la estimulación magnética transcraniana (TMS), lo que será objeto de investigación del GEPECH en un futuro próximo (LEFAUCHEUR et al., 2014).

\section{REFERENCIAS}

ALMEIDA, R. Identificação projetiva, contratransferência e impasse no setting analítico. Revista Mudanças (Universidade Metodista de SP), v. 11, n. 2, p. 213226, 2003.

BATEMAN, A; FONAGY, P. Mentalization based treatment for personality disorders. A practical guide. Oxford, United Kingdom: Oxford University Press, 2016 .

RC: 101104

Disponible: https://www.nucleodoconhecimento.com.br/psicologia-es/neurocienciasaplicada 
BRANDÃO, M. L. et al. Neural organization of different types of fear: Implications for the understanding of anxiety. Revista Brasileira de Psiquiatria, v. 25, n. SUPPL. 2, p. 36-41, 2003.

HOLLIS, J. Os pantanais da Alma: nova vida em lugares sombrios. Toronto. Canadá: Editora Paulus, 1998.

HULLEY, SB; CUMMINGS, SR; BROWNER, WS; GRADY, DG; NEWMAN, T. Delineando a pesquisa clínica: uma abordagem epidemiológica. 3. ed. Porto Alegre: Editora Artimed, 2008.

LEFAUCHEUR, J. P. et al. Evidence-based guidelines on the therapeutic use of repetitive transcranial magnetic stimulation (rTMS). Clinical Neurophysiology, v. 125, n. 11, p. 2150-2206, 2014.

LIEBERMAN, M. D. Social cognitive neuroscience: A review of core processes. Annual Review of Psychology, v. 58, p. 259-289, 2007.

MELLO, P; MONSON, CA; BERTINI, E; COSTA RRPC; MARTINS, MBA; YAMAMOTO, MN; LEITE, J. Neuropsicanálise e a classificação das posições psicanalíticas neokleinianas. Neurociências e Psicologia, v. 13, p. 21-32, 2017.

MEZZASALMA, M. A.; VALENÇA, A. M.; LOPES, F. L.; NASCIMENTO, I.; ZIN, W. A.; NARDI, A. E. Neuroanatomia do transtorno do pânicoRev Bras Psiquiatr 26 (3), , 2004. Disponível em: <http://dx.doi.org/10.1590/S1516-44462004000300010 >

TRINCA, W. Fobia e pânico em psicanálise. São Paulo. Brasil: Editora Vetor Psico-pedagógica Ltda, 1997.

TURATO, E. R. Métodos qualitativos e quantitativos na área da saúde: definições, diferenças e seus objetos de pesquisa. Revista de Saúde Pública, v. 39, n. 3, p. 507-514, 2005. 
YAMADA, M. et al. Induction of galanin after chronic sertraline treatment in mouse ventral dentate gyrus. Brain Research, v. 1516, p. 76-82, 2013.

Enviado: Junio, 2021.

Aprobado: Noviembre, 2021. 